\title{
Informed Consent for Treatment in Forensic Psychiatry: Is It A Realistic Proposition?
}

\author{
Bruno Losier*, Mini Mamak, and Heather Moulden
}

Forensic Psychiatry Program, St. Joseph's Healthcare Hamilton and Department of Psychiatry and Behavioural Neurosciences, McMaster University, Hamilton, Ontario, Canada

*Corresponding Author: Bruno Losier, Forensic Psychiatry Program, St. Joseph's Healthcare Hamilton, West 5th Campus, 100 West 5th, Box 585, Hamilton, ON L8N 3K7, Canada, Tel: 9055221155 Ext. 39357; Email: losierb@stjoes.ca

Received: 09 September 2017; Accepted: 27 September 2017; Published: 02 October 2017

\section{Introduction}

Much has been written with respect to informed consent (IC) in medicine [1]. In his review, Mallardi [2] traces the origins of informed consent to the era of ancient Egyptian, Greek and Roman civilizations. In the beginning, the assumptions of autonomy and what he termed "decisional autodeterminism" of the individual were ill defined and poorly developed. In fact, in those early days, the notion of consent was highly influenced by religious ideology, and the belief that the patient was ignorant in the matters of medicine and its application. Additionally, at the core of this exchange, a universally held notion, that the clinician always acted in the patient's best interest was accepted (a tenant that continues in modern medicine). This belief is embodied in the central tenet of the Hippocratic Oath; that is, 'do no harm'. This promoted a sense of security that the clinician, safeguarding health, had the authority to guide the patient by deciding and choosing what was best for him or her. However, the other unintended consequence of this approach suggested that potential for abuse was great and numerous periods in history have provided irrefutable testimony to that fact, for instance the Tuskegee untreated syphilis project [3]. Over time, as patients became more educated and as new forms of treatment and advancements of novel biotechnology developed, the notion of IC became more complex. In more modern times, emphasis on maintaining dignity and being transparent emerged as central principles of IC, which allowed for greater autonomy on the part of the patient. In general medicine, IC with respect to surgery, diagnostic procedure or prescription of pharmaceutical interventions seems to represent a fairly straightforward endeavor. However, not all healthcare interactions are equal in this respect, particularly where some of the treatments may be non-physical in nature (i.e., psychotherapy), or, in the forensic context, imposed by a 
higher authority (e.g. court). In this perspective, we explore the issue of IC for treatment in forensic settings as an arena for ethical and practical consideration. For our purpose, given the unique issues inherent in treating involuntarily detained individuals who also have significant mental disorders, the focus will remain on treatment specifically within forensic mental health (FMH) settings.

\section{Elements of Informed Consent}

In Canada, the constitution stipulates that health is a provincial matter while criminal law is a federal concern. Consequently, FMH care is regulated by both provincial and federal legislation. The mental health act has evolved immensely since the early 1960s to its current iteration. At its core, the Health Care Consent Act addresses the essential premise of any decision making with respect to healthcare provisions, thus informed consent or IC. As reviewed above IC is a complex application that has received much attention from legal and academic scholars alike. In particular, Applebaum and Gutheil [4] stipulate that IC is comprised of three main principles, which include Information, Voluntariness and Competence. These are briefly described as follows;

1-Information; Sufficient information should be available to the individual to weigh the pros and cons of the proposed procedure or activity. Extensive deliberations over the meaning or significance of 'sufficient' have occurred, and this debate is ongoing to determine if this applies to the clinician, the patient or both. Moreover, discussions also center on the amount and quality of the information; specifically, how much should the clinician share with the patient or how much is required by the patient to truly provide IC. Appelbaum and Gutheil [4] indicate that the "professional standard" seemed to have been the benchmark vs. a radical approach would have the clinician disclose every possible scenario conceivable (albeit unlikely). Although the interest of the informed may dictate a preference for the latter, it would be impractical, and thus the "reasonable person" approach seemed to be a more sensible compromise tailored to the needs of both parties.

2-Voluntariness; The bedrock of voluntariness is sovereignty. IC assumes that once the right amount of information is provided, the individual can either accept or refuse participation without fear of retribution or restriction. However, clinical acumen will support the notion that context can exert great influence on this aspect of IC. Subtle forms of coercion may exist contingent upon the patient-clinician relationship, the fact that the patient may feel a sense of obligation on an inpatient unit given that their basic needs are served (i.e., meals, housing, support, etc.), or disproportionate cooperation with treatment may result in early discharge.

3-Competence; The importance of information and voluntariness can never be understated. If the individual is unable to fully comprehend the scope of what is offered or proposed, then these principles are moot with respect to IC. In other words, no matter how much information is provided and how freely IC is given, if the individual is suspected to lack competence, then the foundation of IC collapses onto itself. Therefore, it must be compulsory for the clinician to determine (i.e., formally assess) how competent the individual with respect to consenting in an informed manner [5]. On the surface, for some, it may be seen as a foregone conclusion on direct observation, although not necessarily an accurate one. 
In the eyes of the law, treatment without consent constitutes battery, while treatment without proper consent is a form of malpractice. Therefore, we need to be sensitive to the various factors that will invariably influence the integrity of IC during our patient interactions. From our perspective there are three primary factors that represent potential pitfalls for IC in forensic settings, namely capacity, competence, and coercion. In a study examining decision making in individuals suffering from mood disorders, Owen and colleagues [6] suggested that manic states constituted a significant risk factor to capacity determination, while mild to moderate depressive symptoms did not seem to have the same impact. However, in more recent times, these authors demonstrated that more severe forms of depression did impact decision making in the context of IC [7]. Additionally, Candia and Barba [8] proposed that although positive symptoms (e.g., hallucination), severity of symptoms, conceptual disorganization, apathy, fluctuating nature of illness, involuntary admission, and lack of insight are strong predictors of incapacity, only 20$30 \%$ of their sample met the incapacity criteria. They cautioned that consent is a fluid process that will vary over time and across diagnostic groups, adding that cognitive dysfunction may be as great a predictor of incapacity as psychopathology.

A further complicating factor is the distinction between understanding and appreciating. Can someone agree to a process/procedure without fully appreciating it? Cea and Fisher [9], in comparing adults with intellectual disability (mild range) with healthy controls, found no difference in terms of choice when considering low-risk elective treatment, while they clearly differed in regards to appreciation and reasoning. More recently, Fisher and colleagues [10] reported that a large proportion of individuals with mild level of intellectual disability and a smaller contingent of individuals with moderate levels of intellectual disability did demonstrate appreciation with respect to IC, even if they ranked lower on the reasoning index component of the assessment when compared to healthy controls. So perhaps competence is not an all or nothing construct, and seemingly varies according to demands, context and diagnostic considerations. Given this knowledge, it behooves the healthcare provider to assess and work systematically (i.e. possibly treat) towards a prompt return to psychiatric stability in order to provide the patient an adequate opportunity to contemplate the elements of the proposed treatment.

\section{The Complexities of Informed Consent in Forensic Psychiatry}

Despite the importance of proper understanding and appreciating of the proposed activities, in many contexts factors that could impact willingness to participate are equally, and possibly more, important. Previous research has focused on a variety of factors that could be construed as subtly and unduly coercive tactics or manipulations (conscious or unconscious). Treatment compliance and response is the mechanism through which symptoms of psychiatric illness are reduced. By virtue of forensic designation, patients in the FMH system, find their risk directly linked to symptoms of their illness, such that the two confer simultaneous weight to the ultimate decision of release. Awareness of this path, from treatment to symptoms, symptoms to risk, and risk to release, patients may experience direct influence or even subtle messages about the relationship between availing themselves of treatment and their eligibility for release and thus their liberty. Under this perspective of FMH, we suggest that true voluntariness might not always be fully, or even partly, achieved.

There is no denying that IC is a complex activity that is quickly mired in static and dynamic variables that include psychopathology status, issues of cognitive capacity, subtle coercion, interpersonal relationships, and context. 
Moreover, there can be no challenge to the fact that in FMH settings the level of complexity and tension within the principles of IC is further intensified. Unlike main stream clinical care, the direct benefit to the patient is not always obvious, may be absent, and, at times, the patient or client may not always be the individual sitting across the table from the healthcare provider (i.e., courts in the adjudication of criminal responsibility). The courts may rule that the patient will be directed to a treatment program as part of sentencing or diversion. In this milieu the three principles of IC are clearly and strongly challenged. Consequently, the clinician could find themselves juggling opposing professional standards or guiding principles of care.

FMH patients are at greater risk for a dual vulnerability. The potential for subtle coercion exists, as it would in any other contexts, but with the added burden of the criminal justice system expectations and oversight on that relationship/activity. In a very complex quagmire of rules and regulations, how freely is the patient able to consider their participation in treatment? Internal and external factors may be at play in influencing decision making in IC. Internally, a patient's personality, past experiences with treatment providers, acceptance of authority, and other factors, can conspire to influence IC. Externally, the influence of legal counsel, the authority of the care provider, and the judiciary can be at odds adding to the complexity of IC. Moreover, the client may perceive coercion when in fact there was none, or there may have been more subtle forms that went undetected by the client. How often do clinicians revisit the patient's understanding of the purpose of the contact, or update the patient on any alterations or deviations from the initial plan? Certainly this has been shown to be critical in other contexts, including being free to refuse participation [11] Consequently, voluntariness, as defined above, we believe is the most tenuous of principles with respect to IC in FMH.

\section{Conclusion}

In the final analysis, the FMH context will always view a measured balance between risk and recovery as paramount. As such, it is imperative that patients have the opportunity to understand and appreciate the value of treatment in a manner that will potentially increase willingness to partake in proposed activities while mitigating the potential for any coercive influence, optimizing full participation and maximal benefit from treatment. Certainly, much has been written with respect to augmenting patient engagement and motivation for change [12], including principles of autonomy as proposed in the theory of self-determination [13]. In this context, the approach would be to focus on the delicate balance (and possible tension) that exists between intrinsic and extrinsic motivational factors. By addressing these issues within the process of IC, we argue that factors that undermine treatment response, such as poor treatment compliance, premature termination or superficial engagement can be reduced. In order to minimize these poor prognostic treatment factors, external rewards are often utilized to maintain FMH patients' interest and engagement in treatment. However, this approach contains a much lower saliency than getting the patient to value the therapeutic progress (internal motivator) as a gateway to improved self-management of one's psychiatric and risk related issues. Thus, central to the idea of voluntariness in FMH patients is a better appreciation of the value of treatment and, therefore, increased willingness to engage in treatment. Although it behooves clinicians to ensure that patients are competent, the patient-clinician relationship will be further strengthened when the possibility of internalizing the benefits of treatment is made more evident. The objective is not to suggest that one approach is better than the next, but simply any strategy is supported in this context (e.g., Motivational Interviewing) insofar as it assists our patients in considering the value of treatment in their ongoing rehabilitation. 
This is not groundbreaking knowledge. This practice is well known to most, if not all, clinicians. Our objective was to provide an opportunity for a simple reminder. So with these considerations in mind we do believe that IC is a realistic proposition in Forensic Psychiatry.

\section{References}

1. Nelson-Marten P, Rich BA. A historical perspective of informed consent In clinical practice and research. Seminars in Oncology Nursing 15 (1999): 81-88.

2. Mallardi V. Le origini del consent informato. Acta Otorhinolaryngol Italia 25 (2005): 312-327.

3. Katz RV, Russell S, Kressin NR, et al. The Tuskegee Legacy Project: willingness of minorities to participate in biomedical research. Journal Health Care Poor Underserved 17 (2006): 698-715.

4. Applebaum PS, Gutheil TG. Malpractice and Other Foprms of Liability. In J. Murphy (Ed), Clinical Handbook of Psychiatry and the Law (4th Edn). Wolters Kluwer, Lippincott Williams \& Wilkins (2010).

5. Appelbaum PS. Assessment of patient's competence to consent to treatment. New England Journal of Medicine 357 (2007): 1834-1840.

6. Owens GS, Richardson G, David AS, et al. Mental capacity to make decisions on treatment in people admitted to psychiatric hospital; cross sectional study. British Medical Journal 337 (2008): a448.

7. Hindmarch T, Hotopf M, Owen GS. Depression and decision making for treatment or research; a systematic review. BioMed Central Medical Ethics 14 (2013): 54-64.

8. Candia PC, Barba AC. Mental capacity and consent to treatment in psychiatric patients: the state of the research. Current Opinion in Psychiatry 24 (2011): 442-446.

9. Cea CD, Fisher CB. Healthcare decision making by adults with mental retardation. Mental Retardation 43 (2003): 78-87.

10. Fisher CB, Cea CD, Davidson PW, et al. Capacity of persons with Mental retardation to consent to participate in randomized clinical trials. American Journal of Psychiatry 160 (2006): 1813-1820.

11. Neff M. Informed Consent; What is it? Who can give it?, How do we improve it? Respiratory Care 53 (2008): 1337-1341.

12. Ryan RM, Lynch MF, Vansteenkiste M, et al. Motivation and Autonomy in Counselling, Psychotherapy and Behavior Change: A look at Theory and Practice. The Counseling Psychologist 39 (2011): 193-260.

13. Deci EL, Ryan RM. The support of autonomy and the control of behavior. Journal of Personality and Social Psychology 53 (1987): 1024-1037.

(C) This article is an open access article distributed under the terms and conditions of the
Creative Commons Attribution (CC-BY) license 4.0 